\title{
The design, development, and implementation of a checklist for intraoperative neuromonitoring changes
}

\author{
John E. Ziewacz, M.D., M.P.H., ${ }^{1}$ Sigurd H. Berven, M.D., ${ }^{2}$ Valli P. Mummaneni, M.D., ${ }^{3}$ \\ Tsung-Hsi Tu, M.D., ${ }^{1,4,5}$ Olaolu C. Akinbo, M.B.B.S., ${ }^{1}$ Russ Lyon, M.S., ${ }^{6}$ \\ and Praveen V. Mummaneni, M.D. ${ }^{1}$ \\ Departments of ${ }^{1}$ Neurosurgery, ${ }^{2}$ Orthopedic Surgery, ${ }^{3}$ Anesthesiology, and ${ }^{6}$ Perioperative Services, \\ University of California, San Francisco, California; ${ }^{4}$ Department of Neurosurgery, Neurological Institute, \\ Taipei Veterans General Hospital; and ${ }^{5}$ School of Medicine, National Yang-Ming University, Taipei, Taiwan
}

Object. The purpose of this study was to provide an evidence-based algorithm for the design, development, and implementation of a new checklist for the response to an intraoperative neuromonitoring alert during spine surgery.

Methods. The aviation and surgical literature was surveyed for evidence of successful checklist design, development, and implementation. The limitations of checklists and the barriers to their implementation were reviewed. Based on this review, an algorithm for neurosurgical checklist creation and implementation was developed. Using this algorithm, a multidisciplinary team surveyed the literature for the best practices for how to respond to an intraoperative neuromonitoring alert. All stakeholders then reviewed the evidence and came to consensus regarding items for inclusion in the checklist.

Results. A checklist for responding to an intraoperative neuromonitoring alert was devised. It highlights the specific roles of the anesthesiologist, surgeon, and neuromonitoring personnel and encourages communication between teams. It focuses on the items critical for identifying and correcting reversible causes of neuromonitoring alerts. Following initial design, the checklist draft was reviewed and amended with stakeholder input. The checklist was then evaluated in a small-scale trial and revised based on usability and feasibility.

Conclusions. The authors have developed an evidence-based algorithm for the design, development, and implementation of checklists in neurosurgery and have used this algorithm to devise a checklist for responding to intraoperative neuromonitoring alerts in spine surgery. (http://thejns.org/doi/abs/10.3171/2012.9.FOCUS12263)

\section{KEY WORDS • $\quad$ surgical checklist $\quad$ algorithm • operating room • neuromonitoring}

$\mathrm{C}$ HECKLISTS have become a routine action item in the aviation field. Over the past few years, general surgeons in several countries have also explored the use of checklists. Since the publication of the WHO surgical safety checklist and the SURPASS checklist in the New England Journal of Medicine in 2009 and 2010, respectively, ${ }^{16,26}$ checklists in surgery have gained a foothold in many surgical departments and operating rooms worldwide. They have even been mandated by the government in certain countries, including the United King-

Abbreviation used in this paper: SURPASS = Surgical Patient Safety System. dom. ${ }^{19,47,50}$ The published study of the WHO checklist implementation demonstrated a decrease in deaths from $1.5 \%$ to $0.8 \%(\mathrm{p}=0.003)$ and a decrease in the overall rate of complications from $11 \%$ to $7 \%(\mathrm{p}<0.001)$ following implementation of the checklist. ${ }^{26}$ The SURPASS study documented exactly the same reduction in mortality $(1.5 \%$ to $0.8 \%, \mathrm{p}=0.003)$ as the WHO study and a reduction in overall complications from $15.4 \%$ to $10.6 \%$ ( $p<0.001)$ following implementation of a perioperative checklist (see Table 1). The combination of these studies led one New England Journal editorialist to suggest that surgical checklists "seem to have crossed the threshold from good idea to standard of care."1 


\section{J. E. Ziewacz et al.}
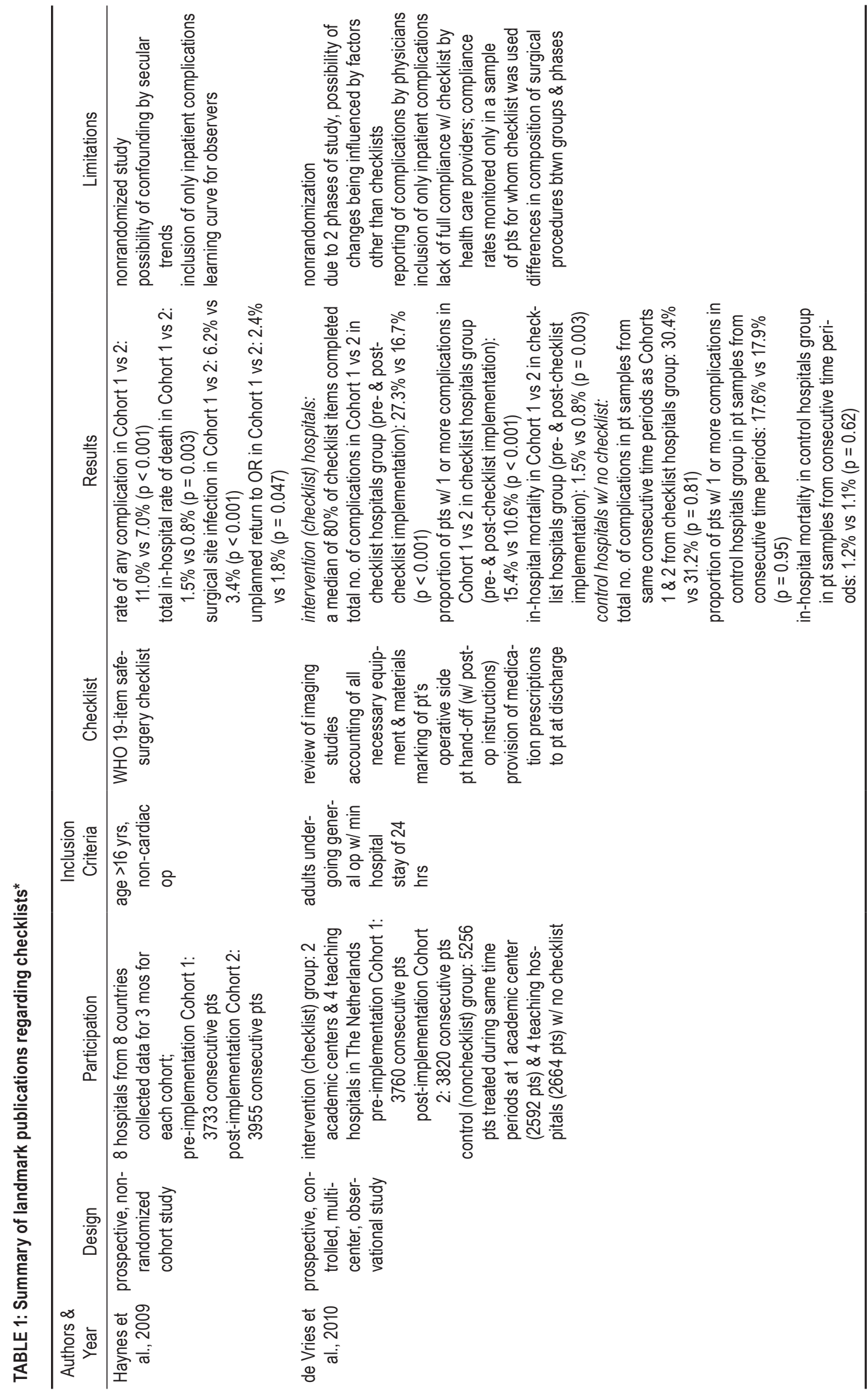

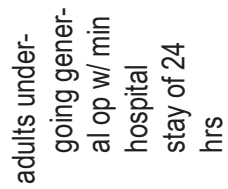
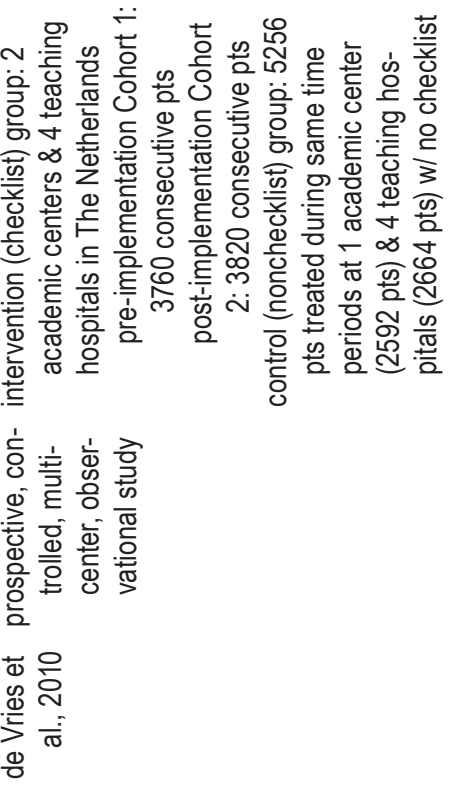
In addition to the reduction of death and complications seen in these studies, checklists may offer other benefits as well. Recent work demonstrates that checklists may reduce costs by preventing complications and may improve team work and patient safety culture. ${ }^{25,42}$ A study in the Netherlands suggested that SURPASS checklist use may reduce malpractice claims..$^{15}$

An area in which the checklist itself may prove most beneficial is during urgent or emergent operations or operating room crises. ${ }^{28,51,54}$ Despite physicians' perception that they are able to perform similarly under emergent and normal circumstances, ${ }^{27,44}$ evidence from the literature suggests that this is not the case. ${ }^{3,5,34,43}$ Surgical emergencies and operating room crises are stressful, time-critical events requiring rapid and coordinated management to provide the best chance of successful outcome. Human performance clearly suffers under this stress and time pressure, ${ }^{34,43}$ and memory aids to combat this decreased performance are largely lacking in the operating room. ${ }^{3,5,30,44}$ Evidence suggests that checklists may be of particular value in these stressful, time-critical situations, when humans are most likely to underperform. ${ }^{28,51,54} \mathrm{~A}$ recent pilot study of a set of crisis checklists for the most common and life-threatening operating room crises demonstrated a 6-fold reduction in surgical teams' failure to adhere to critical steps in management in simulated crises in a high-fidelity simulation setting. ${ }^{54}$ Intraoperative signal loss represents such a situation.

The presence of a new neurological deficit following spine surgery is a rare but problematic complication. ${ }^{38}$ The estimated incidence of a new neurological deficit following spine surgery ranges from $1 \%$ to $1.9 \% .^{24,29,41}$ Multimodality intraoperative neuromonitoring-including monitoring of somatosensory evoked potentials and motor evoked potentials and electromyography-has been shown to be both highly sensitive and specific in detecting a potential neurological deficit..$^{17,18,35}$ The use of intraoperative neuromonitoring allows the surgical team the potential for real-time identification of neural element dysfunction and the possibility to remedy reversible causes, including hypotension, ongoing cord compression, or overcorrection of deformity. ${ }^{21,31,40}$ Due to the strong relationship between anesthetic administration and the effectiveness of monitoring, ${ }^{12-14,36,45,49}$ which can result in false-positive monitoring alerts, ${ }^{32}$ effective teamwork and communication between the anesthesia, neuromonitoring, and surgical teams is necessary to determine the cause and to institute corrective action in response to monitoring alerts. Furthermore, a need for evidencebased protocols for responding to neuromonitoring alerts has been recognized, ${ }^{17}$ and to this end some groups have proposed management protocols for high-risk spine patients that include responses to neuromonitoring changes. ${ }^{23,40}$ In light of the recent evidence demonstrating the potential benefit of checklists in emergent situations, and based on a recognized need to quickly and effectively respond to intraoperative monitoring changes in a cohesive fashion, we sought to develop a checklist for responding to intraoperative monitoring alerts.

Despite the recent momentum behind surgical checklists, checklists are not without their flaws and pit- falls, $, 2,3,9,25,42,47,52$ nor are they simplistic or straightforward to implement. ${ }^{7,47}$ Table 2 depicts the pros and cons associated with surgical checklist use. Evidence from the United Kingdom, France, and Washington State demonstrates significant hurdles to successful checklist adoption., ${ }^{7,19,47}$ Nevertheless, several factors associated with successful checklist implementation have been identified. ${ }^{7}$ Table 3 summarizes barriers to successful checklist implementation and features associated with successful adoption.

Spine surgeons have had relatively little experience incorporating checklists into practice. ${ }^{39}$ Our aims in this paper are to provide a logical, evidence-based algorithm for checklist design, development, and implementation in spine surgery and to provide a checklist for the response to intraoperative neuromonitoring alerts, based on this algorithm.

\section{Methods}

We initially surveyed the literature regarding the key aspects of aviation and surgical checklist design, development, and implementation. ${ }^{3-5,7-11,19,20,22,48,52}$ We focused on the 2 large, prospective studies that were the main impetus for the global spread of the concept of checklists in surgery (Table 1).,16,26 Following this review, we analyzed the benefits and drawbacks of checklists and barriers to successful implementation, as well as successful implementation strategies (Tables 2 and 3). Based on the experience published in the aviation and general surgical literature, we devised an algorithm for the design, development, and implementation of checklists for neurosurgery.

Next, the literature was surveyed for evidence behind best practices for responding to intraoperative monitoring. All stakeholders (neurosurgeons, orthopedic surgeons, anesthesiologists, and neuromonitoring personnel) were then involved in discussions regarding both the content and structure of the checklist. When evidence from the literature for particular items was lacking or contradictory, multidisciplinary consensus was sought to determine the appropriateness of checklist items. An initial draft of the checklist was created, and a small-scale trial was performed with key stakeholders to assess the usability of the checklists. This process was repeated until the usability of the checklist was confirmed. Following these trials, the checklist was revised to its present form.

\section{Results}

\section{An Algorithm for Checklist Design, Development, and Implementation}

We used the recent experience with the design, development, and implementation of checklists in aviation and general surgery to create a logical algorithm for checklist design, development, and implementation in neurosurgery. Figure 1 depicts this algorithm. The key steps include the following: 1) clearly explaining the goals of the project and the philosophy behind it; 2) determining the procedure or condition for which a checklist will be used at an organizational level; 3) obtaining support from the surgical leadership; 4) involving from the outset all stakeholders (surgeons, anesthesiologists, and supporting 
TABLE 2: Checklist pros and cons

\begin{tabular}{|c|c|}
\hline Pros & Cons \\
\hline serve as reminders ensuring critical steps in a procedure are not missed & over-reliance can lead to lack of appropriate use of judgment \& experience \\
\hline can enhance teamwork, communication, culture centered on pt safety & $\begin{array}{l}\text { costs \& resources associated w/ checklist implementation \& outcomes } \\
\text { assessment are barriers to implementation }\end{array}$ \\
\hline $\begin{array}{l}\text { serve as memory aids when humans are most apt to fail (fatigue, stress, } \\
\text { emergencies) }\end{array}$ & $\begin{array}{l}\text { inappropriate checklist selection in an emergency may lead to misman- } \\
\text { agement }\end{array}$ \\
\hline have been shown to reduce death \& complications in other surgical fields & $\begin{array}{l}\text { difficulty achieving appropriate checklist length (too long is onerous \& } \\
\text { impractical, too short may miss critical items) }\end{array}$ \\
\hline initial results in neurosurgery demonstrate error reduction & difficulty obtaining consensus on items to include \\
\hline may reduce net costs by prevention of errors & $\begin{array}{l}\text { overemphasis may divert attention/resources from other safety initiatives } \\
\text { critical to error reduction }\end{array}$ \\
\hline may improve OR efficiency by prevention of errors & $\begin{array}{l}\text { difficulty deciding how many/which procedures will benefit from checklist } \\
\text { use }\end{array}$ \\
\hline may reduce malpractice claims & inability to create checklists for every scenario \\
\hline used in all other high-reliability fields (e.g., & best way to present checklists (paper, electronic, et \\
\hline
\end{tabular}

staff) who will have a role in the use and implementation of the checklist and listening actively to all input; 5) drafting an initial checklist of items (based on evidence review and expert consensus) that are most likely to cause harm if an error of omission or commission occurs (with a focus on keeping the checklist logical and concise as possible); 6) recirculating a draft for review among all stakeholders and redrafting based on multidisciplinary feedback; 7) performing trials in small-scale settings (in a single operating room or in high-fidelity simulation) and redrafting based on usability; 8) training all staff involved in checklist use, focusing on "explaining why" checklists are being implemented and "showing how" they are implemented ${ }^{6}$ (with highly visible involvement of surgical leaders); 9) modifying the checklist to address local hospital considerations; 10) rolling out the checklist initially to early adopters who believe in the concept; 11) rolling out the checklist to the full perioperative staff; 12) measuring compliance and completion to obtain feedback and track outcomes; 13) revising the checklist based on feedback; and 14) providing periodic retraining to accommodate new hires and prevent decay in checklist compliance and completion.

Using this algorithm we created a checklist for intraoperative neuromonitoring alerts (Fig. 2) for use in the surgical treatment of patients with myelopathy (Fig. 3) or spinal deformity (Fig. 4). This checklist includes the items identified through a literature review, which we thought to be critical to properly identifying the cause of an intraoperative monitoring alert and for attempting to reverse those causes. The checklist is meant to be as concise as possible. It is partitioned into sections for the surgeons, anesthesiologists, and neuromonitoring personnel such that they can perform key steps in parallel while ensuring communication is ongoing.

The surgeons are reminded to employ techniques largely aimed at reducing mechanical compression or stretch on the spinal cord. Surgical options include searching for a specific structural cause of compression, such as misplaced hardware or bone graft or releasing deformity correction.

The anesthesia team is directed to review the anesthetic regimen and discuss the anesthetic regimen with the neuromonitoring team to determine possible effects of anesthetic agents on neuromonitoring signal change. Anesthesia options include temporarily withholding any inhalational anesthetic agents (for example, desflurane) and using total intravenous anesthesia. In addition, the anesthesia team is encouraged to discuss the anesthetic regimen with the neuromonitoring personnel and to

\section{TABLE 3: Barriers and keys to successful implementation}

\begin{tabular}{lc}
\hline \multicolumn{1}{c}{ Barriers to Successful Implementation } & Keys to Successful Implementation \\
\hline $\begin{array}{l}\text { lack of engagement of surgical leadership } \\
\text { lack of familiarity w/ checklist use }\end{array}$ & $\begin{array}{c}\text { active \& enthusiastic engagement of surgical \& perioperative lead- } \\
\text { ership } \\
\text { lack of cooperation in OR } \\
\text { inappropriate timing of checks }\end{array}$ \\
$\begin{array}{l}\text { surgical leaders "explaining why" \& "showing how" to use checklist } \\
\text { (lead by example) } \\
\text { inadequate local modification of a multicenter checklist } \\
\text { poor communication btwn anesthesiologists \& surgeons } \\
\text { illogical checklist items }\end{array}$ & $\begin{array}{c}\text { dedicating time to training \& simulation } \\
\text { beginning w/ "early adopters" who are enthusiastic \& able to dem- } \\
\text { ambiguous checklist items } \\
\text { checking off missed checklist items at end of case }\end{array}$ \\
possible pt anxiety due to repeated verbal checks & \\
\hline
\end{tabular}




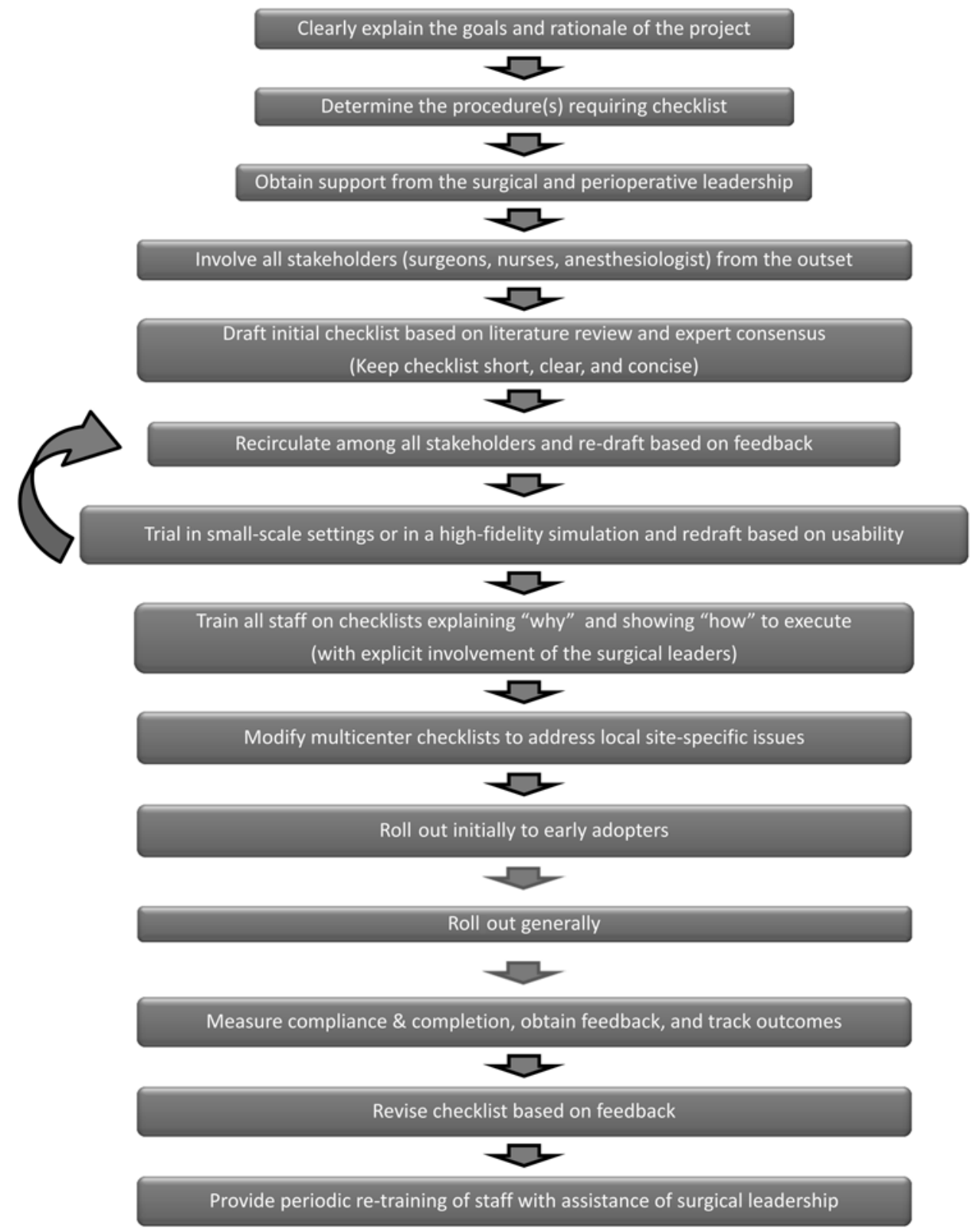

FIG. 1. Algorithm highlighting the key steps for the creation of a neurosurgical checklist.

double-check that the patient has twitches on the twitch monitor to confirm that there is no pharmacological neuromuscular blockade. Furthermore, the anesthesia team may increase the mean arterial pressure in the event that an alert is related to spinal cord hypoperfusion.

The neuromonitoring team is directed to perform actions that largely confirm the change is not due to artifact or equipment malfunction, including repeating the signals and verifying that all leads are correctly placed.

The checklist is designed to enhance teamwork by incorporating checks that specify that team members should communicate key findings and relevant actions to each other. We used the checklist in Fig. 2 in 3 cases with intraoperative neuromonitoring alerts to verify its feasibility. In all 3 cases, the checklist effectively identified anesthetic regimen changes that were responsible for the neuromonitoring alerts. Our team discussed these 3 cases and found that no alterations to the checklist were needed (Fig. 4).

\section{Discussion}

Previous efforts to reduce errors in medicine and surgery have not met with the same success as efforts at error prevention in aviation and other high-reliability fields. ${ }^{26,30,53}$ Checklists for surgery are being pursued due to the increasing recognition that errors in surgery are possible, may result in harm, and may be preventable. The experience with checklists in surgery draws heavily from the aviation industry.

The experience in the aviation industry and initial surgical experience with checklists in normal and 


\section{Checklist for Neuromonitoring (MEP) Alert in Patients with Myelopathy or Deformity}

\section{Spine Surgeon:}

Stop current manipulation

Assess field for structural cord compression (misplaced hardware or bone graft, osteophytes, or hematoma)

Perform further decompression if stenosis is present

Consider reversing correction of a spinal deformity

\section{Neurophysiologist:}

Repeat trials of MEPs and SSEPs to rule out potential false positive

Check all leads to make sure no pull-out, may add leads in proximal muscle

groups if possible

Assess the pattern of changes

Asymmetric changes (associated with cord or nerve root injury)

Symmetric changes (associated with anesthetic or hypotension issues)

Quantify improvement and communicate to the surgical team

\section{Anesthesiologist:}

Check if neuromuscular blockade (muscle relaxant) given

If yes, $\square$ Check train of four (TOF)

Verify that no change in anesthetic administration occurred

Assess anesthetic depth

BP $\square$ RR $\square$ HR $\square$ BIS monitor (if available)

Restore or maintain blood pressure (goal mean arterial pressure of 90-100)

Check Hemoglobin/Hematocrit (goal hemoglobin >9-10)

Check temperature and I/O's for adequate resuscitation

Check extremity position in case of plexus palsy

Lighten depth of anesthesia

Reduce to $1 / 3$ MAC or temporarily eliminate inhaled agents (i.e. desflurane)

Reduce intravenous anesthetics such as propofol (which may accumulate

systemically during the case and blunt MEPs)

Add adjuvant agents such as Ketamine to permit reduction of MEP

suppressive agents (i.e. propofol and inhalational anesthetics)

\section{If No Change:}

Increase MAP >100

Consider Steroid Administration

Consider Wake-up test

Consider Aborting surgery

Consider Calcium Channel Blocker (topical to cord or iv)

*The checklist assumes baseline anesthetic regimen is 1/3-1/2 MAC of halogenated anesthetic (desflurane) and TIVA

(total intravenous anesthesia) with propofol +/- ketamine.

FIG. 2. Checklist for the response to an intraoperative neuromonitoring alert. $B I S=$ bispectral index; $B P=$ blood pressure; $H R$ = heart trate; $\mathrm{I} / \mathrm{O}=$ input/output; $\mathrm{MAC}=$ minimum alveolar concentration; $\mathrm{MAP}=$ mean arterial pressure; $\mathrm{MEP}=$ motor evoked potential; $\mathrm{RR}=$ respiration rate; $\mathrm{SSEP}=$ somatosensory evoked potential.

emergent settings demonstrates that they can engender cultural change and improve teamwork and communication. ${ }^{16,25,26,54}$ It is for these reasons, as well as for potential corollary benefits such as reducing malpractice claims and generating cost savings,,${ }^{15,42}$ that neurosurgeons have begun to explore the utility of checklists.

Before embarking on checklist use, however, neurosurgeons must be aware of significant potential drawbacks 

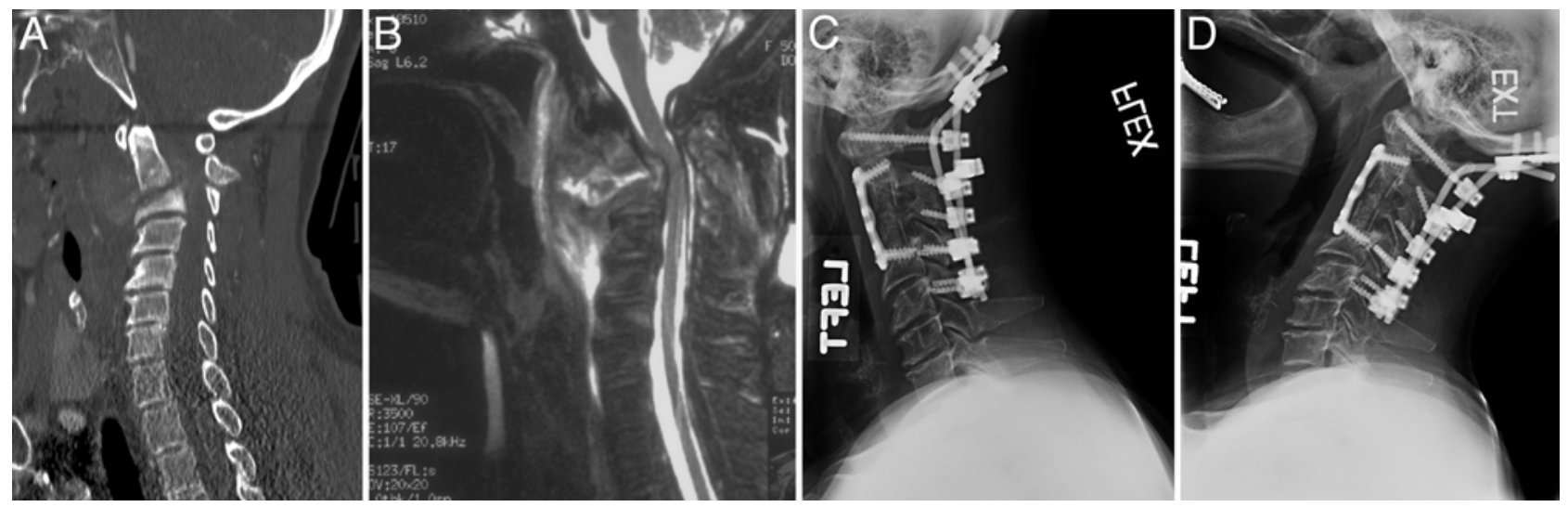

FIG. 3. Illustrative surgical case involving a patient with myelopathy in whom intraoperative neuromonitoring was used. A: Preoperative cervical spine CT sagittal reconstruction demonstrating cervical kyphotic deformity with a high risk for intraoperative neurological deficit. B: Preoperative cervical sagittal T2-weighted MR image demonstrating cervical kyphosis, stenosis, and cord signal change at the C-2 level. C: Flexion lateral cervical spine radiograph obtained after circumferential cervical decompression and stabilization. D: Extension lateral cervical spine radiograph obtained after circumferential cervical decompression and stabilization.
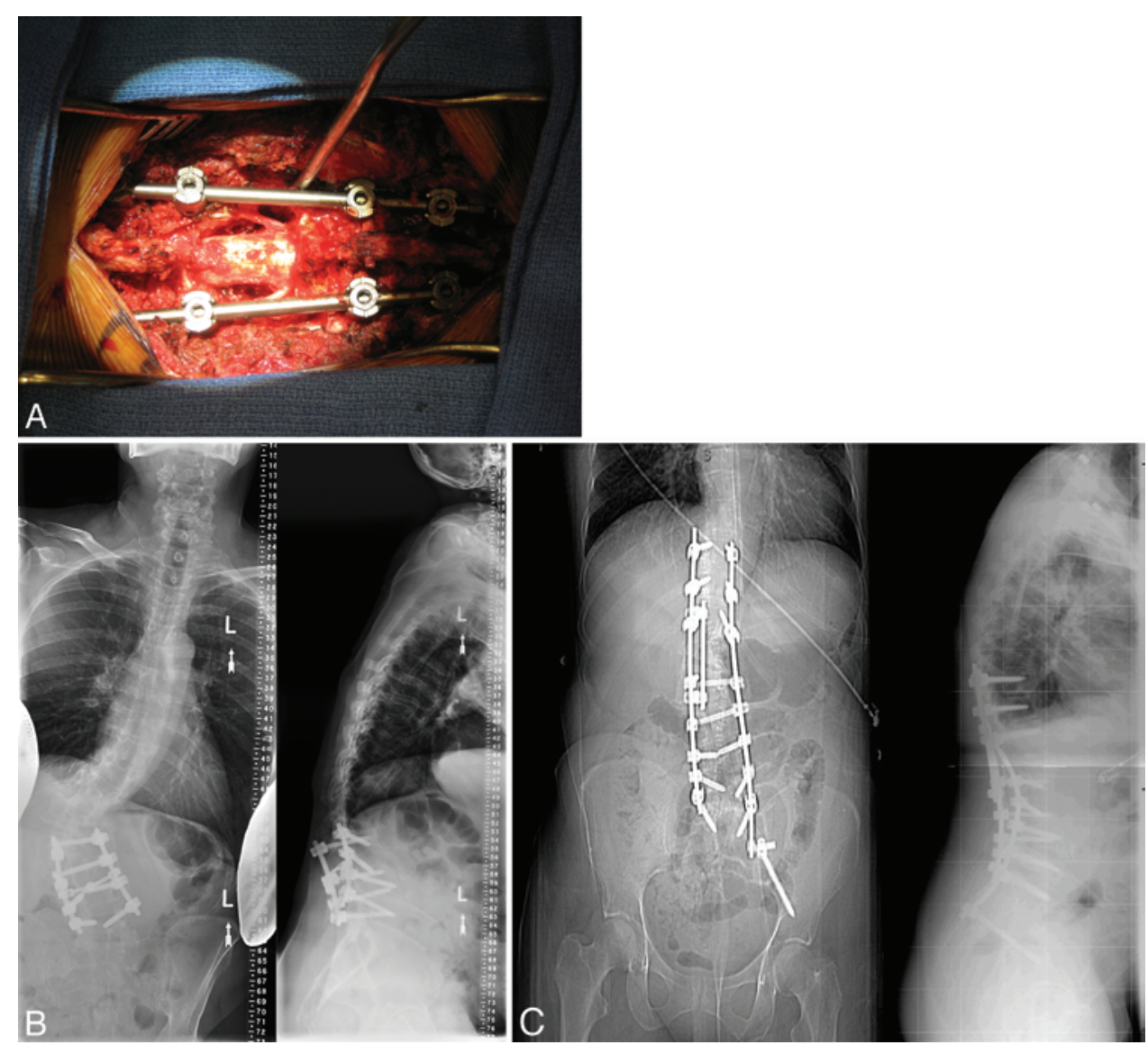

FIG. 4. Illustrative surgical case involving a patient with spinal deformity who was at risk for an intraoperative neurological deficit due to the magnitude of the correction required at the level of the conus medullaris. The surgery was performed with intraoperative neuromonitoring. The checklist was used in this case during an intraoperative neuromonitoring alert. Use of the checklist revealed that a change to the anesthetic regimen altered the motor evoked potential signals. A: Intraoperative photograph showing a pedicle subtraction osteotomy at the level of the conus (L-1). B and C: Preoparative (B) and postoperative (C) radiographs demonstrating correction of coronal and sagittal deformity and imbalance. 
and pitfalls. Checklists are not a panacea, nor do they substitute for a culture oriented toward patient safety. Especially, they are never a replacement for sound judgment, solid training, and personal responsibility. Furthermore, checklists do not exist in a void. They are implemented into specific social and cultural contexts ${ }^{19}$ that vary by profession, location, department, and operating room. In the case of surgical checklists, they often are employed in contexts that have been historically hostile to notions of safety initiatives due to their potential hindrance of a practitioner's autonomy. ${ }^{28}$ Checklists have at times been viewed by practitioners as a bureaucratic nuisance that is employed just to meet accreditation standards. ${ }^{50}$ Given this, it is not surprising that implementation of checklists, particularly the WHO checklist, has met resistance and has not achieved complete penetrance, even in locations in which it is mandated..$^{7,19,47,50}$ Calland et al. ${ }^{6}$ studied the implementation of a surgical checklist by randomly assigning 10 surgeons to checklist use or no checklist use for performance of laparoscopic cholecystectomies. Video review of surgical teams that employed the checklist demonstrated a significant increase in positive safety-related team behaviors, such as explicit communication of roles and responsibilities, contingency planning, and postsurgical debriefings. However, subjective ratings of cases by surgeons showed that those in the checklist arm consistently felt their cases involved less effective communication and less team efficiency than those in the nonchecklist arm. ${ }^{6}$ This highlights the fact that even if objective evidence supports the benefits of checklists in their cases, surgeons may be reluctant to implement checklists, given their subjective attitudes toward them. Nevertheless, some efforts at implementation have been remarkably successful. 7,37 The Department of Neurological Surgery at the Mayo Clinic in Arizona achieved a 99.5\% compliance rate over 8 years with a checklist for use prior to neurosurgical procedures. ${ }^{37}$ In his article describing the experience, Lyons ${ }^{37}$ notes that checklist and other safety and quality improvement initiatives are readily implemented at that institution, in part due to a surgical culture dedicated to patient safety where checklist adoption is believed to add value to patient care and peer pressure is exerted to bring surgeons into compliance with patient safety initiatives. Accordingly, Conley et al. ${ }^{7}$ studied the implementation of the WHO checklist in Washington State and noted that successful implementation depended heavily on an institution's surgical culture, and in particular, the enthusiastic engagement of surgical leaders in "explaining why" and "demonstrating how" checklists could be effective." We placed these factors at the top of our algorithm.

Based on the knowledge that human cognitive capacity declines in stressful and time-critical situations, groups have begun to devise checklists for emergency situations, with some initial promise. ${ }^{28,51,54}$ A pilot study of a group of checklists for the 12 most common and life-threatening operating room crises in a high-fidelity simulator found that failure of surgical teams to adhere to known best practices for management was reduced 6-fold when checklists were used to manage the crises. ${ }^{54}$ Along these lines, neurosurgeons have begun to tailor checklists to their specific needs, ${ }^{33}$ including emergent situations. ${ }^{46}$ Recognizing the time-dependent and stressful nature of intraprocedural aneurysmal rupture, as well as the complexity of coordinating the multiple steps in appropriate management, Taussky and colleagues ${ }^{46}$ have developed a checklist for endovascular intraprocedural aneurysm rupture. Loss of intraoperative monitoring signals represents a similarly time-critical, stressful situation, with an array of causes involving surgical, anesthetic, and technical possibilities-both benign and potentially catastrophic, and it requires a rapid, coordinated approach to the identification and resolution of the causal elements. Because of this multidisciplinary management complexity and the time-critical nature of an effective response, intraoperative neuromonitoring alerts may be the type of situation most conducive to checklist use.

Previous work has sought to provide a framework for addressing responses to intraoperative neuromonitoring signal loss. Pahys and colleagues, ${ }^{40}$ in reviewing neurological injury in the treatment of idiopathic scoliosis, provide an algorithm for an approach to neuromonitoring signal loss that includes many factors critical to management in such instances, including raising the mean arterial pressure, checking the patient's hemoglobin level, and releasing corrective forces. Their algorithm, however, is not presented in simple checklist form, which may limit its utility in the time-critical scenario encountered in the event of intraoperative monitoring signal change. In detailing their comprehensive protocol for managing high-risk spine patients at Northwestern, Halpin et al. ${ }^{23}$ highlighted the intraoperative features necessary for appropriate management, especially the need for communication among teams and keeping up with blood loss. This management protocol, however, is meant to encompass the full scope of care of high-risk spine patients from their preoperative evaluation through their postoperative course. $^{23}$ This broad and comprehensive protocol may not be ideally suited for the rapid response needed in the event of intraoperative neuromonitoring signal change.

Our neuromonitoring alert checklist is brief, straightforward to use, and has been effective in our experience. It involves the key stakeholders in surgeries for spinal myelopathy or spinal deformity (the surgeons, the anesthesia team, and the neuromonitoring team). It highlights the key items that often lead to neuromonitoring alerts and provides rapid steps to assess and remedy correctable issues.

Despite the promise of a checklist to guide the response to an intraoperative neuromonitoring alert, many questions remain. The checklist has not yet undergone a large-scale trial to assess generalizable utility. The ideal person to read and run the checklist (for example, the surgeon, anesthesiologist, or monitoring specialist) is unknown, and the choice is not straightforward, given that many tasks must occur simultaneously. Similarly the best presentation of the checklist, whether it be on the wall, on the anesthesia machine, or accessible in electronic form, for instance, is unknown. Finally, the clinical efficacy is not known. To demonstrate the clinical efficacy of a checklist for intraoperative neuromonitoring alert, a large, multicenter trial would likely be necessary to demonstrate a measurable change in outcome given the relatively low incidence of neurological injury. ${ }^{10,13,20}$ 


\section{Conclusions}

Checklists have long been used in aviation to reduce error, and checklists in general surgery have recently demonstrated efficacy in reducing complications and death. This has led some neurosurgeons to begin utilizing checklists with encouraging initial results. However, neurosurgeons and orthopedic surgeons must be mindful of the drawbacks and limitations of checklists. Surgeons should remember that checklists are never a substitute for sound clinical judgment, good training, and personal responsibility. We have created an algorithm for design, development, and implementation of a spine surgeon's checklist and have used this algorithm to create a checklist for the multidisciplinary response to significant intraoperative neuromonitoring alerts. The algorithm for checklist design may be used to create checklists for other intraoperative issues. Ideally, neurosurgeons and orthopedic surgeons should create and implement neurosurgical checklists rather than have them dictated by others.

\section{Disclosure}

Dr. Praveen Mummaneni is a past consultant for DePuy Spine. $\mathrm{He}$ receives other financial support (royalties) from DePuy Spine, Quality Medical Publishers, and Thieme Medical Publishers; and travel support from Globus Medical. Dr. Valli Mummaneni is Dr. Praveen Mummaneni's spouse, but has no additional disclosures. Dr. Berven receives honoraria from Alphatec, DePuy, Globus, Medtronic, and Stryker. He receives other financial support (royalties) from Medtronic. He owns stock in AccuLIF, Baxano, and DTRAX.

Author contributions to the study and manuscript preparation include the following. Conception and design: all authors. Acquisition of data: Ziewacz, Berven, Lyon. Analysis and interpretation of data: Ziewacz, Berven, Lyon. Drafting the article: Tu, Ziewacz, Akinbo. Critically revising the article: Tu, Ziewacz, Berven, VP Mummaneni, Akinbo, PV Mummaneni. Reviewed submitted version of manuscript: all authors. Approved the final version of the manuscript on behalf of all authors: Tu. Administrative/technical/ material support: PV Mummaneni. Study supervision: PV Mummaneni.

\section{References}

1. Birkmeyer JD: Strategies for improving surgical qualitychecklists and beyond. N Engl J Med 363:1963-1965, 2010

2. Bosk CL, Dixon-Woods M, Goeschel CA, Pronovost PJ: Reality check for checklists. Lancet 374:444-445, 2009

3. Burian BK: Aeronautical emergency and abnormal checklists: expectations and realities, in Proceedings of the Human Factors and Ergonomics Society 50th Annual Meeting 50. San Francisco, 2006

4. Burian BK: Design guidance for emergency and abnormal checklists in aviation, in Proceedings of the Human Factors and Ergonomics Society 50th Annual Meeting. San Francisco, 2006

5. Burian BK, Dismukes RK, Barshi I: The emergency and abnormal situations project, in McCarthy T (ed): Proceedings of the ISASI 2003 Conference. August 26-28, 2003. Washington, DC

6. Calland JF, Turrentine FE, Guerlain S, Bovbjerg V, Poole GR, Lebeau K, et al: The surgical safety checklist: lessons learned during implementation. Am Surg 77:1131-1137, 2011

7. Conley DM, Singer SJ, Edmondson L, Berry WR, Gawande AA: Effective surgical safety checklist implementation. J Am Coll Surg 212:873-879, 2011

8. Degani A: On the typography of flight-deck documentation.
NASA Technical Memorandum \#177605. Moffett Field, CA: NASA Ames Research Center, 1992

9. Degani A, Wiener EL: Cockpit checklists: concepts, design, and use. Hum Factors 35:28-43, 1993

10. Degani A, Wiener EL: On the design of flight-deck procedures. NASA Technical Memorandum \#177642. Moffett Field, CA: NASA Ames Research Center, 1994

11. Degani A, Wiener EL: Procedures in complex systems: the airline cockpit. IEEE Trans Syst Man Cybern A Syst Hum 27: 302-312, 1997

12. Deiner S: Highlights of anesthetic considerations for intraoperative neuromonitoring. Semin Cardiothorac Vasc Anesth 14:51-53, 2010

13. Deletis V, Sala F: Intraoperative neurophysiological monitoring of the spinal cord during spinal cord and spine surgery: a review focus on the corticospinal tracts. Clin Neurophysiol 119:248-264, 2008

14. Devlin VJ, Anderson PA, Schwartz DM, Vaughan R: Intraoperative neurophysiologic monitoring: focus on cervical myelopathy and related issues. Spine J 6 (6 Suppl):212S-224S, 2006

15. de Vries EN, Eikens-Jansen MP, Hamersma AM, Smorenburg SM, Gouma DJ, Boermeester MA: Prevention of surgical malpractice claims by use of a surgical safety checklist. Ann Surg 253:624-628, 2011

16. de Vries EN, Prins HA, Crolla RM, den Outer AJ, van Andel $\mathrm{G}$, van Helden SH, et al: Effect of a comprehensive surgical safety system on patient outcomes. N Engl J Med 363:19281937,2010

17. Fehlings MG, Brodke DS, Norvell DC, Dettori JR: The evidence for intraoperative neurophysiological monitoring in spine surgery: does it make a difference? Spine 35 (9 Suppl):S37S46, 2010

18. Feng B, Qiu G, Shen J, Zhang J, Tian Y, Li S, et al: Impact of multimodal intraoperative monitoring during surgery for spine deformity and potential risk factors for neurological monitoring changes. J Spinal Disord Tech 25:E108-E114, 2012

19. Fourcade A, Blache JL, Grenier C, Bourgain JL, Minvielle E: Barriers to staff adoption of a surgical safety checklist. BMJ Qual Saf 21:191-197, 2012

20. Gawande A, Boorman D, Brighan and Women's Hospital Center for Surgery and Public Health Dissemination Team: A checklist for checklists. Project Check Website. (http://www. projectcheck.org/checklist-for-checklists.html) [Accessed September 20, 2012]

21. Gonzalez AA, Jeyanandarajan D, Hansen C, Zada G, Hsieh PC: Intraoperative neurophysiological monitoring during spine surgery: a review. Neurosurg Focus 27(4):E6, 2009

22. Hales B, Terblanche M, Fowler R, Sibbald W: Development of medical checklists for improved quality of patient care. Int $\mathbf{J}$ Qual Health Care 20:22-30, 2008

23. Halpin RJ, Sugrue PA, Gould RW, Kallas PG, Schafer MF, Ondra SL, et al: Standardizing care for high-risk patients in spine surgery: the Northwestern high-risk spine protocol. Spine (Phila Pa 1976) 35:2232-2238, 2010

24. Hamilton DK, Smith JS, Sansur CA, Glassman SD, Ames CP, Berven SH, et al: Rates of new neurological deficit associated with spine surgery based on 108,419 procedures: a report of the scoliosis research society morbidity and mortality committee. Spine (Phila Pa 1976) 36:1218-1228, 2011

25. Haynes AB, Weiser TG, Berry WR, Lipsitz SR, Breizat AH, Dellinger EP, et al: Changes in safety attitude and relationship to decreased postoperative morbidity and mortality following implementation of a checklist-based surgical safety intervention. BMJ Qual Saf 20:102-107, 2011

26. Haynes AB, Weiser TG, Berry WR, Lipsitz SR, Breizat AH, Dellinger EP, et al: A surgical safety checklist to reduce morbidity and mortality in a global population. N Engl J Med 360:491-499, 2009 
27. Helmreich RL: On error management: lessons from aviation. BMJ 320:781-785, 2000

28. Hunter DN, Finney SJ: Follow surgical checklists and take time out, especially in a crisis. BMJ 343:d8194, 2011

29. Kamerlink JR, Errico T, Xavier S, Patel A, Patel A, Cohen A, et al: Major intraoperative neurologic monitoring deficits in consecutive pediatric and adult spinal deformity patients at one institution. Spine (Phila Pa 1976) 35:240-245, 2010

30. Karl RC: Aviation. J Gastrointest Surg 13:6-8, 2009

31. Kelleher MO, Tan G, Sarjeant R, Fehlings MG: Predictive value of intraoperative neurophysiological monitoring during cervical spine surgery: a prospective analysis of 1055 consecutive patients. J Neurosurg Spine 8:215-221, 2008

32. Kim DH, Zaremski J, Kwon B, Jenis L, Woodard E, Bode R, et al: Risk factors for false positive transcranial motor evoked potential monitoring alerts during surgical treatment of cervical myelopathy. Spine (Phila Pa 1976) 32:3041-3046, 2007

33. Kramer DR, Halpern CH, Connolly PJ, Jaggi JL, Baltuch GH: Error reduction with routine checklist use during deep brain stimulation surgery. Stereotact Funct Neurosurg 90:255259, 2012

34. Kurrek MM, Devitt JH, Cohen M: Cardiac arrest in the OR: how are our ACLS skills? Can J Anaesth 45:130-132, 1998

35. Li F, Gorji R, Allott G, Modes K, Lunn R, Yang ZJ: The usefulness of intraoperative neurophysiological monitoring in cervical spine surgery: a retrospective analysis of 200 consecutive patients. J Neurosurg Anesthesiol 24:185-190, 2012

36. Lotto ML, Banoub M, Schubert A: Effects of anesthetic agents and physiologic changes on intraoperative motor evoked potentials. J Neurosurg Anesthesiol 16:32-42, 2004

37. Lyons MK: Eight-year experience with a neurosurgical checklist. Am J Med Qual 25:285-288, 2010

38. Malhotra NR, Shaffrey CI: Intraoperative electrophysiological monitoring in spine surgery. Spine (Phila Pa 1976) 35:21672179,2010

39. McConnell DJ, Fargen KM, Mocco J: Surgical checklists: a detailed review of their emergence, development, and relevance to neurosurgical practice. Surg Neurol Int 3:2, 2012

40. Pahys JM, Guille JT, D'Andrea LP, Samdani AF, Beck J, Betz RR: Neurologic injury in the surgical treatment of idiopathic scoliosis: guidelines for assessment and management. J Am Acad Orthop Surg 17:426-434, 2009

41. Pastorelli F, Di Silvestre M, Plasmati R, Michelucci R, Greggi $\mathrm{T}$, Morigi A, et al: The prevention of neural complications in the surgical treatment of scoliosis: the role of the neurophysiological intraoperative monitoring. Eur Spine J 20 (1 Suppl 1):S105-S114, 2011

42. Semel ME, Resch S, Haynes AB, Funk LM, Bader A, Berry WR, et al: Adopting a surgical safety checklist could save money and improve the quality of care in U.S. hospitals. Health Aff (Millwood) 29:1593-1599, 2010
43. Semeraro F, Signore L, Cerchiari EL: Retention of CPR performance in anaesthetists. Resuscitation 68:101-108, 2006

44. Sexton JB, Thomas EJ, Helmreich RL: Error, stress, and teamwork in medicine and aviation: cross sectional surveys. BMJ 320:745-749, 2000

45. Sloan TB, Heyer EJ: Anesthesia for intraoperative neurophysiologic monitoring of the spinal cord. J Clin Neurophysiol 19:430-443, 2002

46. Taussky PH, Lanzino G, Cloft H, Kallmes D: A checklist in the event of aneurysm perforation during coiling. AJNR Am J Neuroradiol 31:E59, 2010

47. Vats A, Vincent CA, Nagpal K, Davies RW, Darzi A, Moorthy $\mathrm{K}$ : Practical challenges of introducing WHO surgical checklist: UK pilot experience. BMJ 340:b5433, 2010

48. Verdaasdonk EG, Stassen LPS, Widhiasmara PP, Dankelman $\mathrm{J}$ : Requirements for the design and implementation of checklists for surgical processes. Surg Endosc 23:715-726, 2009

49. Wang AC, Than KD, Etame AB, La Marca F, Park P: Impact of anesthesia on transcranial electric motor evoked potential monitoring during spine surgery: a review of the literature. Neurosurg Focus 27(4):E7, 2009

50. Warnock GL: Of surgeons and safety checklists. Can J Surg 53:364-366, 2010

51. Weiser TG, Haynes AB, Dziekan G, Berry WR, Lipsitz SR, Gawande AA: Effect of a 19-item surgical safety checklist during urgent operations in a global patient population. Ann Surg 251:976-980, 2010

52. Weiser TG, Haynes AB, Lashoher A, Dziekan G, Boorman DJ, Berry WR, et al: Perspectives in quality: designing the WHO Surgical Safety Checklist. Int J Qual Health Care 22:365-370, 2010

53. Zegers M, de Bruijne MC, de Keizer B, Merten H, Groenewegen PP, van der Wal G, et al: The incidence, root-causes, and outcomes of adverse events in surgical units: implication for potential prevention strategies. Patient Saf Surg 5:13, 2011

54. Ziewacz JE, Arriaga AF, Bader AM, Berry WR, Edmondson L, Wong JM, et al: Crisis checklists for the operating room: development and pilot testing. J Am Coll Surg 213:212-217, e10, 2011

Manuscript submitted July 16, 2012.

Accepted September 18, 2012.

Please include this information when citing this paper: DOI: 10.3171/2012.9.FOCUS12263.

Address correspondence to: Tsung-Hsi Tu, M.D., Department of Neurosurgery, University of California, San Francisco, 505 Parnassus Avenue, Room M-780, San Francisco, California 94143-0332. email: thtu0001@gmail.com. 